\title{
PASCOLO A ROTAZIONE IN BRACHIARIA BRIZANTHA CV. MARANDU PER MIGLIORARE LA PRODUZIONE DI LATTE
}

\section{ARTICOLO ORIGINALE}

SOARES, Marcio Groto ${ }^{1}$, CAMARGO, Simone Cristina², FRASSETTO, Matheus Orlandin ${ }^{3}, \mathrm{ABREU}$, Hélio Mar de ${ }^{4}$

SOARES, Marcio Groto. Et al. Pascolo a rotazione in Brachiaria brizantha cv. marandu per migliorare la produzione di latte. Revista Científica Multidisciplinar Núcleo do Conhecimento. Anno. 06, Ed. 09, Vol. 07, pp. 104-118. Settembre 2021. ISSN: 2448-0959, Link di accesso: https://www.nucleodoconhecimento.com.br/veterinaria-it/pascolo-a-rotazione, DOI: 10.32749/nucleodoconhecimento.com.br/veterinaria-it/pascolo-a-rotazione

\section{RIEPILOGO}

Nelle proprietà lattiero-casearie, affinché ci sia un aumento del profitto, è necessario aumentare l'efficienza dei fattori di produzione, in particolare la nutrizione, che incide sul costo fisso. Si cerca sempre più un equilibrio tra la fornitura di mangime e il pascolo. II metodo di pascolo rotazionale è stato adottato da diversi produttori, in quanto ha una buona produttività in un'area più piccola. Tuttavia, i pascoli utilizzati in questo tipo di pascolo, pur avendo una qualità eccellente, hanno fabbisogni nutrizionali più elevati. L'obiettivo di questo lavoro era quello di effettuare un'analisi economica del costo della produzione di latte, attraverso l'impianto di pascolo a rotazione, e lo scambio di pascolo autoctono per Brachiaria brizantha $c v$. marandu, in una fattoria di famiglia. Questo lavoro è stato preparato nella città di Foz do Iguaçu. È stata utilizzata un'area di $6300 \mathrm{~m}^{2}$, suddivisa in 28 paddock di $225 \mathrm{~m}^{2}$ utilizzando

\footnotetext{
${ }^{1}$ Laureato.

2 Dottorato.

${ }^{3}$ Insegnante.

${ }^{4}$ Laureato.
}

RC: 99522

Disponibile in: https://www.nucleodoconhecimento.com.br/veterinaria-it/pascolo-arotazione 
un recinto elettrico. II costo totale di produzione è stato di $R \$ 6.047,40$, con un margine positivo di $\mathrm{R} \$ 5.143,61$ all'anno. In base ai risultati ottenuti nelle analisi economiche, si conclude che l'impianto di pascolo a rotazione e lo scambio di pascolo autoctono per Brachiaria brizantha $c v$. Marandu è una gestione valida, poiché il reddito generato dalla vendita del latte era sufficiente a coprire i costi totali di produzione.

Parole chiave: Costo, Latte, Foraggio, Picchetto, Manipolazione.

\section{INTRODUZIONE}

II Brasile è al quarto posto nella classifica mondiale della produzione di latte. Nel 2018, la regione meridionale ha rappresentato il 34\% della produzione totale del paese (ANUÁRIO LEITE, 2019). II solo stato del Paraná quest'anno ha prodotto una media di 4,37 miliardi di litri di latte, di cui il Paraná occidentale era responsabile del 19\% (IBGE, 2018).

I bovini da latte nello stato del Paraná sono un'attività tipica dell'agricoltura familiare, che rappresenta circa l'85,1\% dei produttori dello stato (SEAB, 2018). La maggior parte di questi produttori utilizza il pascolo come principale fonte di cibo per gli animali. La superficie media occupata con pascoli nello stato è di 16,6 ettari (IPARDES, 2009).

Sebbene il Brasile si distingua nella produzione di latte, la sua produttività è di circa $1.689 \mathrm{~L} /$ mucca / anno, che è inferiore a quella di Stati Uniti, Cina, Russia, Argentina, Unione Europea e Nuova Zelanda, che producono più di 3 L / mucca / anno. Uno dei fattori responsabili della bassa produzione di latte è l'uso di erbe scarsamente produttive e di bassa qualità nutrizionale, insieme a una gestione inadeguata sia degli animali che dei pascoli (GONÇALVES et al., 2003).

Un modo per intensificare la produzione animale nei sistemi di pascolo è l'adozione di foraggi migliorati, più adatti al clima, con un maggiore potenziale produttivo e una

RC: 99522

Disponibile in: https://www.nucleodoconhecimento.com.br/veterinaria-it/pascolo-arotazione 
migliore qualità (JANK, 2017). Tra i vari foraggi esistenti in Brasile, spicca la varietà ibrida di erba Brachiaria brizantha cv. marandu, per presentare un buon valore nutrizionale e un'elevata produzione di biomassa, adattandosi bene a terreni di media e buona fertilità, essendo tollerante all'acidità del suolo (FONSECA et al., 2010).

La corretta gestione del pascolo dovrebbe essere un altro punto da osservare nelle proprietà dei bovini da latte. Uno dei pascoli che si è dimostrato praticabile è la rotazione, dove c'è una suddivisione del pascolo in tre o più picchetti. Questo tipo di pascolo presenta diversi vantaggi, quali: maggiore uniformità di pascolo, maggiore utilizzo di foraggi, maggiore tasso di allevamento e maggiore longevità delle inesprenze che formano ciuffi (FUKUMOTO et al., 2010).

Diversi studi hanno mostrato buoni risultati per i bovini da latte, con l'impianto del pascolo rotazionale nell'erba di Brachiaria brizantha cv. marandu (GONÇALVES, 2003); (DEMSKI, 2013), (MOURA, 2017). Secondo (ANDRADE, 2008), questo tipo di pascolo non tollera il pascolo continuo.

Con la corretta scelta del foraggio, una corretta gestione e un migliore utilizzo dell'area di proprietà, cerca di generare risparmi per il produttore, poiché ridurrà i costi di produzione. Secondo (GERON, 2012), il costo di produzione è la somma di tutti gli importi spesi per le operazioni e i servizi insumand utilizzati nel processo di produzione di una determinata attività. Con lo studio dei costi, è possibile verificare come vengono remunerate le risorse utilizzate in un processo produttivo e come è la redditività dell'attività (GERON et al., 2014).

Pertanto, l'obiettivo di questo lavoro era quello di eseguire l'analisi economica del costo della produzione di latte, attraverso l'implementazione del pascolo rotazionale e lo scambio di pascoli autoctoni per pascoli di Brachiaria brizantha cv. marandu, in una tenuta agricola familiare.

RC: 99522

Disponibile in: https://www.nucleodoconhecimento.com.br/veterinaria-it/pascolo-arotazione 


\section{MATERIALI E METODI}

L'esperimento è stato condotto in un caseificio situato nella città di Foz do Iguaçu -

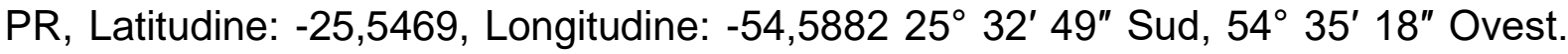
Questa proprietà comprende una superficie di 5,0 ettari, in questa zona si è sviluppato un allevamento tradizionale da latte della regione, dove gli animali avevano accesso all'intera area di pascolo, composta da erba autoctona a bassa produttività, non vi era disponibilità di sale minerale, per gli animali in lattazione era disponibile solo sale comune nell'abbeveratoio e solo un'integrazione di $10 \mathrm{~kg}$ di mungitura e l'accesso all'acqua avveniva da un ruscello che attraversa la proprietà.

La proprietà dispone di sei bovini da latte, composti da quattro mucche adulte, due mucche in allattamento e due mucche essiccate, oltre a due giovenche.Questi animali sono stati gestiti in tutta l'area di pascolo durante tutto l'anno e gli animali in allattamento hanno ricevuto $10 \mathrm{~kg}$ di mangime industrializzato al giorno.

I lavori sono iniziati con una visita alla proprietà per una diagnosi. Le misurazioni dell'area di pascolo sono state eseguite utilizzando il GPS (Figura 1) e anche la raccolta del suolo (Figura 2). I risultati dell'analisi del suolo sono (Tabella 1).

RC: 99522

Disponibile in: https://www.nucleodoconhecimento.com.br/veterinaria-it/pascolo-arotazione 
Figura 1 - Foto satellitare dell'area sperimentale, con la delimitazione dell'area di pascolo:

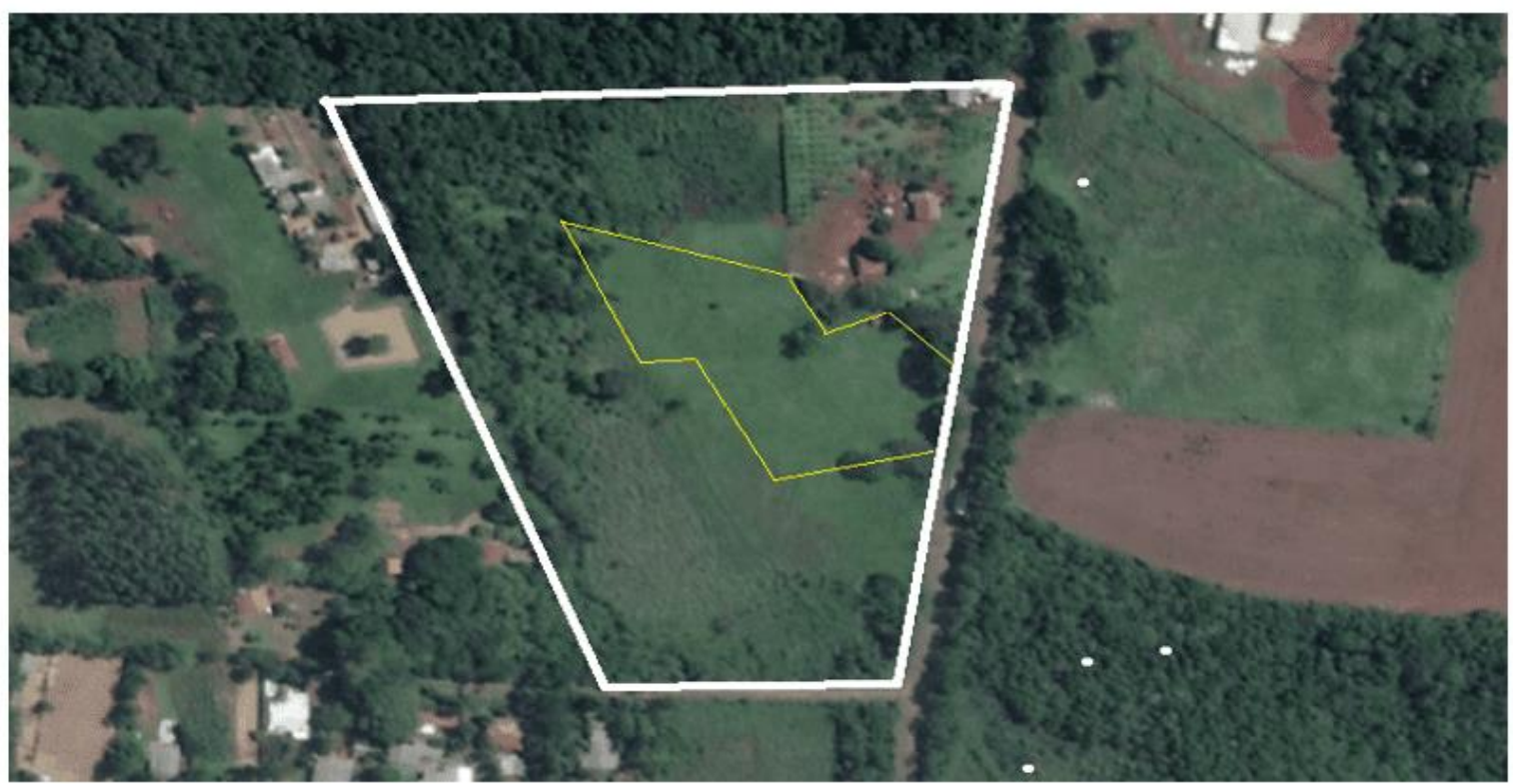

Fonte: Google Earth.

Figura 2 - Raccolta di campioni di terreno:

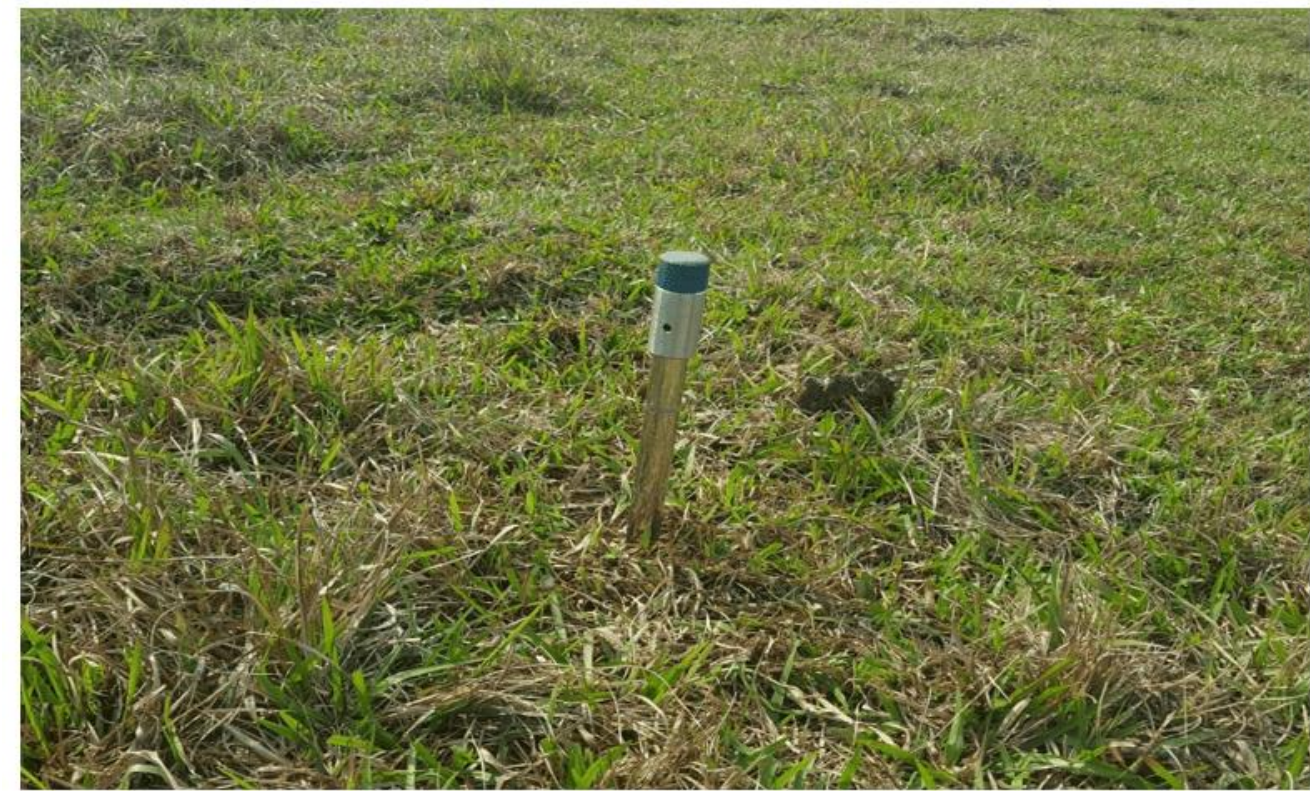

Fonte: Archivio personale.

RC: 99522

Disponibile in: https://www.nucleodoconhecimento.com.br/veterinaria-it/pascolo-arotazione 
Tabella 1 - Risultati dell'analisi del suolo dell'area sperimentale:

\begin{tabular}{|c|c|c|c|c|c|c|c|c|c|c|c|c|c|c|}
\hline $\mathrm{pH}$ & & & $\mathrm{K}$ & $\mathrm{Ca}$ & $\mathrm{Mg}$ & $\mathrm{Al}$ & $\mathrm{H}+\mathrm{Al}$ & CTC & SB & $\mathrm{V}$ & $\mathrm{m}$ & $\mathrm{Ca}$ & $\mathrm{Mg}$ & $\mathrm{K}$ \\
\hline \multirow[t]{2}{*}{$\mathrm{CaCl} 2$} & M.O & $\mathrm{P}$ (res) & & & & & & & & & & & & \\
\hline & $\mathrm{g} / \mathrm{Kg}$ & $\mathrm{mg} / \mathrm{dm} 3$ & \multicolumn{5}{|c|}{$\mathrm{mmol} / \mathrm{dm} 3$} & $\%$ & & & \multicolumn{4}{|c|}{ \% CTC } \\
\hline 2 & 38 & 14 & 3 & 21 & 5 & 2,7 & 29 & 58 & 29 & 50 & 8 & 36 & 9 & 5 \\
\hline
\end{tabular}

Fonte: FZEA/USP.

Dopo aver ricevuto l'analisi del suolo, sono stati effettuati i calcoli di correzione del suolo, dove il V\% era del $58 \%$. Per correggere il $\mathrm{pH}$ del terreno sono stati necessari $800 \mathrm{~kg} \mathrm{ha}^{-1}$ di calcare dolomitico con l'86\% di PRNT. Per correggere il fosforo è stato necessario aggiungere $250 \mathrm{~kg} \mathrm{ha}^{-1}$ di semplice fertilizzante perfosfato. Per la concimazione produttiva sono stati utilizzati $250 \mathrm{~kg} \mathrm{ha}^{-1}$ di urea agricola (FRASSETO, 2015).

Per iniziare la preparazione del terreno, l'applicazione del calcare è stata applicata in tutta l'area sperimentale e, poco dopo, è stata utilizzata una griglia pesante, con una profondità di $40 \mathrm{~cm}$, che è servita a incorporare il calcare nel terreno, rompere le compattazioni superficiali e dare condizioni alla crescita delle nuove specie foraggi.

Per un dimensionamento dell'area necessaria per il pascolo, le mucche sono state pesate attraverso un nastro di pesatura con una precisione del 95\%, per un totale di $1.950 \mathrm{~kg} \circ 4,5 \mathrm{UA}$. A causa del fatto che sono stati utilizzati bovini da latte, è stato determinato che gli animali avrebbero avuto accesso a un nuovo picchetto alla fine di una giornata di pascolo. Pertanto, sono stati utilizzati i dati di (FRASSETO 2015), in cui un UA (450 kg di peso vivo) richiede almeno $50 \mathrm{~m}^{2}$ al giorno di area di pascolo. Sapendo che c'erano 4,5 UA, l'area di ogni picchetto era di $225 \mathrm{~m}^{2}$. Sono stati costruiti un totale di 28 picchetti, utilizzando 0,63 ettari. Durante la costruzione dei picchetti, è stato necessario aggiungere tre corridoi, larghi $2 \mathrm{~m}$.

RC: 99522

Disponibile in: https://www.nucleodoconhecimento.com.br/veterinaria-it/pascolo-arotazione 
Per lo spacco dei picchetti sono stati utilizzati 50 mourões, 72 tondo per cemento armato di $10 \mathrm{~mm}, 32$ tondo per cemento armato di $12 \mathrm{~mm}, 500 \mathrm{~m}$ di filo tri zincato per circa il perimetro totale dell'area e $2500 \mathrm{~m}$ di filo elettroplastico per la divisione interna dei picchetti, 150 isolatori di tipo marrone, 22 isolatori a gancio e 114 isolatori tipo tondo per cemento armato. II filo metallico con un'altezza di $80 \mathrm{~cm}$ è stato utilizzato in relazione al terreno e ogni $10 \mathrm{~m}$ è stato impiantato un tondo da $1,5 \mathrm{~m}$ con isolante per mantenere l'altezza in relazione al terreno, il filo elettroplastico è stato utilizzato anche per dividere i picchetti e ridurre i costi di formazione. Per elettrificare il filo, è stato utilizzato un elettrificatore Sentinel modello 30.000 , con un minimo di 5000 volt.

Figura 3 - Schizzo della divisione del picchetto:

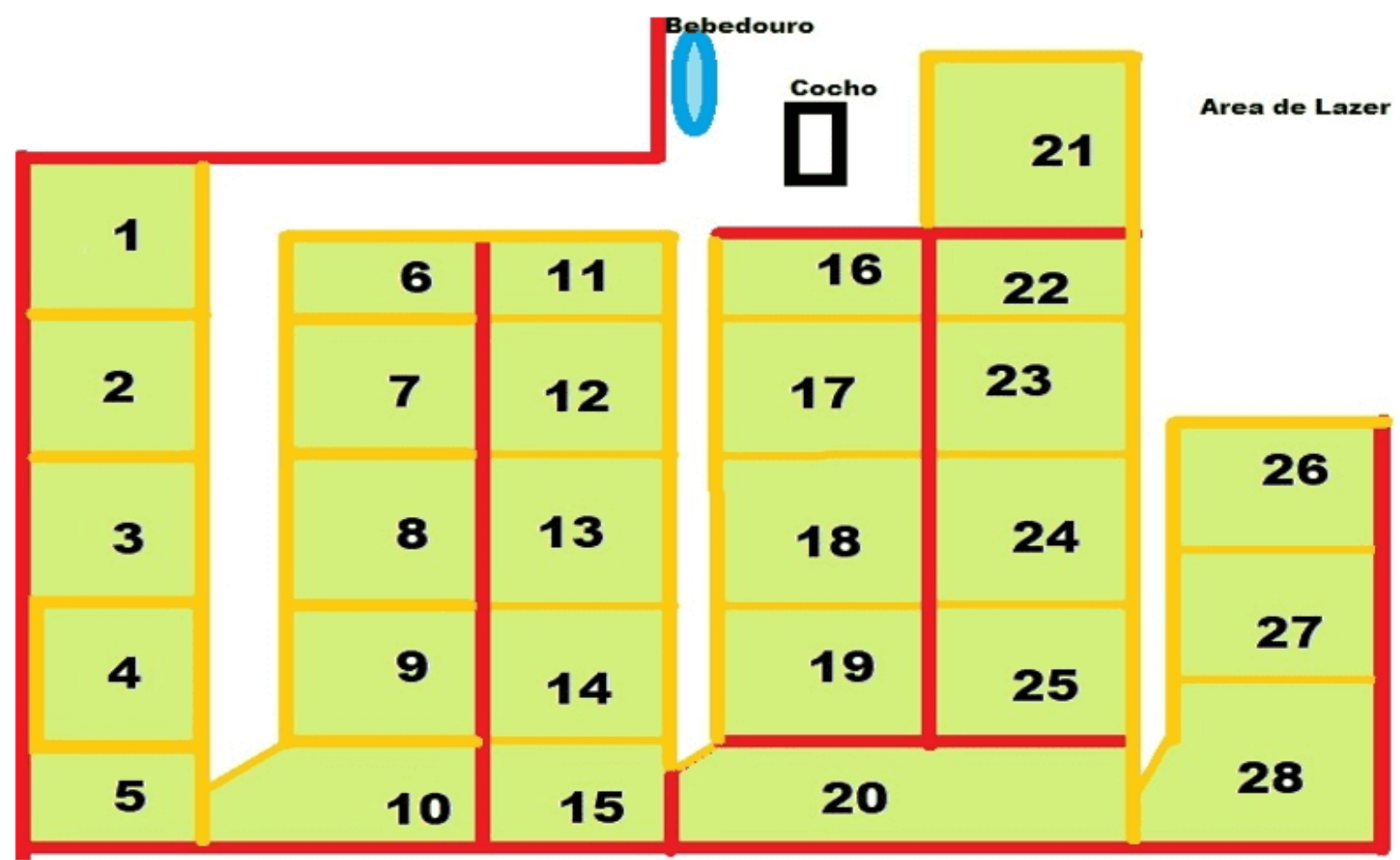

Fonte: Archivio personale.

Il foraggio scelto è stato Brachiaria Brizantha $c v$. marandu, la gestione di questo foraggio inizia quando la pianta raggiunge i $30 \mathrm{~cm}$ in entrata e $15 \mathrm{~cm}$ in uscita

RC: 99522

Disponibile in: https://www.nucleodoconhecimento.com.br/veterinaria-it/pascolo-arotazione 
Andrade (2008); Fonseca (2010). La semina del foraggio e la fertilizzazione delle piante sono iniziate il 15/11/2019, con il metodo di semina a spaglio e l'incorporazione di semi e fertilizzante fosfatico super semplice in seguito. I semi sono stati incrostati, utilizzando $15 \mathrm{~kg} \mathrm{ha}^{-1}$.

La prima concimazione produttiva è stata effettuata 60 giorni dopo la semina, applicando $125 \mathrm{~kg} \mathrm{ha}^{-1}$ di urea agricola con il $45 \%$ di azoto. II primo pascolo è iniziato il 02/03/2020, 77 giorni dopo la semina. La seconda concimazione della produzione applicando $125 \mathrm{Kg} \mathrm{ha}^{-1}$ di urea agricola è stata effettuata nel primo ciclo di pascolo essendo 28 giorni dopo l'inizio del primo pascolo.

Per il resto degli animali è stato aggiunto un bevitore d'acqua con circa $100 \mathrm{~L}$ di acqua, aggiunta di sale minerale adatto per bovini da latte e gli alberi della proprietà stessi servivano da ombreggiamento.

Per eseguire l'analisi economica e la valutazione del costo di produzione del latte, abbiamo identificato gli agenti coinvolti, nonché i valori che rappresentano gli effetti dell'implementazione del progetto su questi agenti, considerando anche la chiusura di un anno.

Per valutare il costo di attuazione del progetto, abbiamo considerato i costi di investimento nella preparazione del suolo e nella semina dell'erba, nonché gli investimenti per infrastrutture e attrezzature nella formazione di picchetti.

Per valutare il costo di produzione, abbiamo utilizzato la struttura del costo operativo di produzione, proposta da Matsunaga (1976). In cui, il costo operativo effettivo (COE), corrisponde alla spesa di denaro da parte del produttore, con l'acquisto di mangimi, le spese per il mantenimento di pascoli e medicinali, per un anno.

Per il costo totale di produzione (CTP), l'ammortamento è stato aggiunto a (COE). L'ammortamento annuale del pascolo è stato calcolato dalla somma delle forniture

RC: 99522

Disponibile in: https://www.nucleodoconhecimento.com.br/veterinaria-it/pascolo-arotazione 
necessarie per la costruzione della recinzione elettrica suddivise in un periodo di dieci anni. Ammortamento del 10\% annuo (CANZIANI et al., 2000).

Per calcolare le entrate, sono state considerate le entrate nette, che è l'importo guadagnato nella consegna del latte all'anno meno i costi totali di produzione (MARTIN, 1997).

\section{RISULTATI E DISCUSSIONE}

All'interno della produzione agricola, è necessario avere una pianificazione da parte del produttore, in modo che ci sia crescita e sviluppo dell'attività. Pertanto, il primo passo nello studio della fattibilità di un progetto è quello di rilevare le spese per la sua attuazione. I costi per l'attuazione di questo progetto, dalla preparazione del terreno per la semina del pascolo, alla formazione dei picchetti sono stati $R \$$ 3,023.70.

Prima di eseguire l'impianto dell'erba $B$. brizantha cv. marandu, era necessario preparare il terreno. Gli investimenti relativi alla lavorazione del suolo sono descritti in (Tabella 2).

Tabella 2 - Costi di investimento nella preparazione e nella semina del suolo:

\begin{tabular}{|l|l|l|l|}
\hline Descrizione & Unità & Quantità & Valore $(\mathrm{R} \$)$ \\
\hline Analisi del terreno & Uni & 1 & 33,00 \\
\hline $\begin{array}{l}\text { Calcare dolomitico (PRNT } \\
86 \%)\end{array}$ & $\mathrm{Kg}$ & 800 & 156,00 \\
\hline Perfosfato (00.19.00) & $\mathrm{Kg}$ & 250 & 287,46 \\
\hline Tempo di arazione del trattore & $\mathrm{H}$ & 2 & 400,00 \\
\hline Seme incrostato & $\mathrm{Kg}$ & 15 & 287,01 \\
\hline Urea Agricola & $\mathrm{Kg}$ & 250 & 400,00 \\
\hline Erbicida Glifosato & $\mathrm{Ml}$ & 500 & 35,00 \\
\hline
\end{tabular}

RC: 99522

Disponibile in: https://www.nucleodoconhecimento.com.br/veterinaria-it/pascolo-arotazione 


\begin{tabular}{|l|l|l|l|}
\hline Olio 2T & Ml & 250 & 20,00 \\
\hline Carburante - Benzina & L & 5 & 20,00 \\
\hline Totale & & & $\mathbf{1 . 6 3 8 , 4 7}$ \\
\hline
\end{tabular}

Fonte: Dati di progetto.

I costi di lavorazione del terreno e di semina hanno rappresentato il $54,20 \%$ dei costi totali per l'impianto dei pascoli. Le pratiche correttive e la fertilizzazione del suolo sono solo una parte dei requisiti necessari per il successo del sistema di produzione del pascolo (PEREIRA et al., 2018).

È importante che il produttore investa ed esegua una buona preparazione del terreno e una corretta implementazione del pascolo, cercando sempre l'equilibrio nel sistema suolo-pianta-animale (PEREIRA et al., 2018). Queste gestione influenzeranno l'aumento della produzione animale, nonché la riduzione delle spese per i mangimi per animali.

Nella ricerca di una corretta gestione del pascolo, è stato suddiviso in picchetti. Gli investimenti per infrastrutture e attrezzature nella formazione di picchetti sono rappresentati in (tabella 3).

Tabella 3 - Tabella dei costi per la formazione di picchetti, con la recinzione elettrica:

\begin{tabular}{|l|l|l|l|}
\hline Descrizione & Unità & Quantità & Valore (R\$) \\
\hline Filo Eletrix & $\mathrm{m}$ & 500 & 184,00 \\
\hline Filo elettroplastico 500 m & $\mathrm{m}$ & 5 & 104,82 \\
\hline Isolante marrone & Uni & 150 & 176,98 \\
\hline $\begin{array}{l}\text { Isolante per cemento } \\
\text { armato }\end{array}$ & Uni & 114 & 131,65 \\
\hline $\begin{array}{l}\text { Isolante a gancio corto } \\
\text { Isolante a gancio lungo }\end{array}$ & Uni & 20 & 24,00 \\
\hline
\end{tabular}

RC: 99522

Disponibile in: https://www.nucleodoconhecimento.com.br/veterinaria-it/pascolo-arotazione 


\begin{tabular}{|c|c|c|c|}
\hline $\begin{array}{l}\text { Interruttore } \\
\text { commutazione }\end{array}$ & Uno & 3 & 36,00 \\
\hline Barrato (3/8") 1,5 m & Uno & 72 & 284,30 \\
\hline Barra (1/2") 1,5 m & Uno & 2 & 186,18 \\
\hline Tubo pvc 20 mm 6 m & Uno & 1 & 12,80 \\
\hline Voltmetro digitale & Uno & 1 & 139,90 \\
\hline Elettrificatore & Uno & 1 & 100,00 \\
\hline Totale & & & $1.385,23$ \\
\hline
\end{tabular}

Fonte: Dati di progetto.

I costi delle infrastrutture e delle attrezzature per la formazione dei picchetti rappresentano il $45,80 \%$ dei costi totali della realizzazione del pascolo. La gestione razionale del foraggio ha ampiamente dimostrato i benefici della divisione dei pascoli, dove c'è una maggiore uniformità di pascolo, un maggiore utilizzo dei foraggi, un più alto tasso di allevamento e una maggiore longevità delle erbe in erba che formano il ciuffo (FUKUMOTO, 2010), che è il caso di B. brizantha cv. marandu. L'uso della recinzione elettrica, nelle sue diverse forme nel decapaggio, è uno strumento necessario per implementare a basso costo le suddivisioni che il pascolo rotazionale richiede (EMBRAPA, 1999).

I beni che compongono l'impresa sono soggetti a costanti svalutazioni, principalmente a causa dell'usura e dell'invecchiamento. (Tabella 4) ha i dati di ammortamento della recinzione elettrica.

Tabella 4 - Ammortamento di materiali e attrezzature:

\begin{tabular}{|l|l|l|l} 
Descrizione & Importo totale & Periodo di validità & Valore \\
$\mathrm{R} \$$ & & (anni) & annuo
\end{tabular}

RC: 99522

Disponibile in: https://www.nucleodoconhecimento.com.br/veterinaria-it/pascolo-arotazione 
Costruzione di recinzioni $1.385,25$

elettriche

Fonte: Dati di progetto.

Tabella 5 - Pagamento degli investimenti:

\begin{tabular}{|l|l|l|l|l|}
\hline Descrizione & $\begin{array}{l}\text { Importo totale } \\
\mathrm{R} \$\end{array}$ & $\begin{array}{l}\text { Tempo di pagamento } \\
\text { (anni) }\end{array}$ & $\begin{array}{l}\text { Valore } \\
\text { annuo }\end{array}$ \\
\hline $\begin{array}{l}\text { Costo } \\
\text { implementazione }\end{array}$ & di & $\mathbf{3 , 0 2 3 . 7 0}$ & $\mathbf{1 0}$ & 302.37 \\
\hline
\end{tabular}

Fonte: Dati di progetto.

Per avere un maggiore controllo annuale sulla gestione aziendale, gli investimenti per la distribuzione sono stati suddivisi in 10 anni. 4,20\% delle entrate annue derivanti dalla vendita di latte, descritte in (Tabella 5).

Per calcolare l'assunzione monetaria, sotto forma di denaro in un anno, è stato considerato l'importo pagato al produttore per litro di latte, venduto quotidianamente e direttamente dal produttore del modulo per un anno. Per calcolare la quantità di latte annuale è stata considerata una mungitura giornaliera al mattino con un totale di 10 litri / giorno (Tabella 6).

Tabella 6 - Entrate della vendita annuale di latte:

\begin{tabular}{|l|l|}
\hline Variabili & Valore totale \\
\hline Prezzo al litro $(\mathrm{R} \$)$ & 2,00 \\
\hline Litri totali di latte prodotti (L) & $3.600,00$ \\
\hline
\end{tabular}

RC: 99522

Disponibile in: https://www.nucleodoconhecimento.com.br/veterinaria-it/pascolo-arotazione 
Fonte: Dati di progetto.

Ogni anno il produttore dovrà effettuare una spesa di denaro, che è considerato un costo operativo effettivo (COE). Sono stati presi in considerazione i costi dei mangimi, le spese per il mantenimento del pascolo e dei farmaci (tabella 7).

Tabella 7 - Costo operativo effettivo - COE, all'anno:

\begin{tabular}{|l|l|l|l|l|}
\hline Descrizione & Unità & Quant & $\begin{array}{l}\text { Valore unitario } \\
\mathrm{R} \$\end{array}$ & $\begin{array}{l}\text { Importo totale } \\
\mathrm{R} \$\end{array}$ \\
\hline Razione & $\mathrm{Kg}$ & 720 & 1,32 & 950,04 \\
\hline Urea agricola & $\mathrm{Kg}$ & 250 & 1,60 & 400,00 \\
\hline Energia elettrica & $\mathrm{R} \$$ & 12 & 0,60 & 216,00 \\
\hline Vaccino contro la brucellosi & Dose & 6 & 1,62 & 9,72 \\
\hline Vaccino contro la rabbia & Dose & 6 & 1,48 & 8,88 \\
\hline Dectomax 50 ml & Uni. & 1 & 21,90 & 21,90 \\
\hline $\begin{array}{l}\text { Vaccino contro l'afta } \\
\text { epizootica }\end{array}$ & Dose & 6 & 1,50 & 9,00 \\
\hline Totale & & & & \\
\hline
\end{tabular}

Fonte: Dati di progetto.

Si osserva in questo lavoro che la maggiore spesa per la produzione è per i mangimi. Secondo (MARTINEZ, 2009), la spesa per la nutrizione rappresenta in media il 67\% dei costi di produzione del latte in Brasile. Pertanto, si cercano strategie per ridurre l'uso di diete e altri integratori. Migliorare il pascolo e gestirlo è un'alternativa che aiuta la produzione di latte a essere economicamente sostenibile. Per questo motivo, il pascolo rotazionale è stato sempre più indicato (MARION et al., 2010).

RC: 99522

Disponibile in: https://www.nucleodoconhecimento.com.br/veterinaria-it/pascolo-arotazione 
Rosestolato (2015), analizzando la redditività economica della produzione di latte di due proprietà distinte per quanto riguarda il sistema tecnologico di produzione, ha osservato che il (COE) sulla proprietà in cui le mucche erano tenute in un sistema di pascolo continuo e integrato con mangimi concentrati e sale minerale era superiore a quello mantenuto in un sistema di pascolo rotazionale e integrato con canna da zucchero tritata, mangimi concentrati e sali minerali.

Nella proprietà valutata in questo studio, sono stati somministrati $10 \mathrm{~kg}$ di mangime industrializzato per animale in allattamento e dopo l'implementazione del progetto c'è stata una diminuzione di $9 \mathrm{~kg}$ iniziando a offrire solo $1 \mathrm{~kg}$ di mangime agli animali, diminuendo così il (COE), come osservato da Rosestolato (2015). Tuttavia, l'integrazione agli animali anche con il cambiamento del sistema di pascolo è importante, poiché aiuta a fornire negli animali $\mathrm{i}$ nutrienti che mancano nella composizione del foraggio. II costo del mangime rappresentava il $44,70 \%$ di tutti i costi di produzione.

Considerando i valori di ammortamento e di costo operativo effettivo (COE), il costo totale di produzione è stato ottenuto in un anno (Tabella 8).

Tabella 8 - Costo totale di produzione - CTP (base annua):

\begin{tabular}{|l|l|}
\hline Descrizione & Importo totale R $\$$ \\
\hline Deprezzamento & 138,52 \\
\hline Costi COE & $1.615,50$ \\
\hline $\begin{array}{l}\text { Pagamento } \\
\text { dell'investimento }\end{array}$ & 302.37 \\
\hline Totale & $\mathbf{2 . 0 5 6 , 3 9}$ \\
\hline
\end{tabular}

Fonte: Dati di progetto.

Nei paesi con bassi prezzi del latte, i produttori sono in grado di ridurre il costo di produzione aumentando la partecipazione del pascolo alla dieta delle vacche da

RC: 99522

Disponibile in: https://www.nucleodoconhecimento.com.br/veterinaria-it/pascolo-arotazione 
latte. In questo studio, il costo totale di produzione rappresentava il $28,55 \%$ del reddito annuo.

Per calcolare le entrate, sono state considerate le entrate nette, che è il valore guadagnato nella consegna del latte all'anno meno i costi totali di produzione (tabella 9).

Tabella 9 - Entrate (su base annua):

\begin{tabular}{|l|l|}
\hline Descrizione & Importo totale R\$ \\
\hline Ricavo & $7.200,00$ \\
\hline $\begin{array}{l}\text { Costo totale di produzione(CTP su base } \\
\text { annua) }\end{array}$ & $2.056,39$ \\
\hline Bilancia & $\mathbf{5 . 1 4 3 , 6 1}$ \\
\hline
\end{tabular}

Fonte: Dati di progetto.

Secondo Oliveira (2007), la conoscenza delle implicazioni dell'effettivo costo operativo (COE), nel breve periodo, è fondamentale nella gestione del business, e il margine lordo deve essere positivo, se negativo, si raccomanda l'interruzione della produzione.

L'analisi finanziaria dell'attuazione di questo progetto si è rivelata fattibile (tabella 9). Alla luce delle analisi effettuate sulla base dei dati ottenuti, è stato osservato che l'investimento nella tecnica del pascolo a rotazione nei bovini da latte presenta un rendimento economicamente sostenibile del capitale investito e che la stessa influenza positiva sulla produzione rende questa attività più competitiva nel settore. Quindi questa tecnica consente la potenziale espressione per la produzione di latte e l'aumento della redditività dei produttori, che renderebbe redditizia l'attività lattierocasearia, anche in tempi di calo del prezzo del latte.

RC: 99522

Disponibile in: https://www.nucleodoconhecimento.com.br/veterinaria-it/pascolo-arotazione 


\section{CONSIDERAZIONI FINALI}

Secondo i risultati ottenuti nelle analisi economiche, si conclude che l'implementazione del pascolo rotazionale e lo scambio di pascoli autoctoni da parte di Brachiaria brizantha cv. marandu, è una gestione praticabile, poiché i ricavi generati dalla vendita di latte erano sufficienti a coprire i costi totali di produzione.

Questo sistema di produzione semi-intensiva è consigliato alla realtà locale, perché è un'alternativa di innovazione tecnologica, in grado di ridurre le spese con l'acquisto di mangimi, e fornire una buona performance animale, dal cambio di gestione del pascolo e all'inserimento dell'erba di migliore valore nutrizionale e maggiore produzione di massa.

\section{RIFERIMENTI}

ANDRADE, Carlos Mauricio Soares de. Pastejo Rotacionado: Tecnologia para Aumentar a Produtividade de Leite e a Longevidade das Pastagens. EMBRAPA - Acre, dez. 2008.

ANUÁRIO LEITE 2019. Sua excelência, o consumidor: novos produtos e novas estratégias da cadeia do leite para ganhar competitividade e conquistar os clientes finais. Brasília, DF: Texto Comunicação Corporativa, 2019. Disponível em: <https://www.infoteca.cnptia.embrapa.br/infoteca/handle/doc/1109959>. Acesso em: 25 mar. 2020.

DEMSKI, Joana Baptista. Desempenho e comportamento de vacas lactantes em pastagens de cultivares de braquiárias. Dissertação apresentada ao Programa de Pós-graduação do Instituto de Zootecnia, APTA/SAA. Nova Odessa - SP, 2013. $80 \mathrm{p}$.

FONSECA, Delermando Miranda da; MARTUSCELLO, Janaina Azevedo. Plantas forrageiras. Viçosa, 537p. 2010.

RC: 99522

Disponibile in: https://www.nucleodoconhecimento.com.br/veterinaria-it/pascolo-arotazione 
FUKUMOTO, Nelson Massaru; DAMASCENO, Júlio Cesar; DERESZ, Fermino, MARTINS, Carlos Eugênio; CÓSER, Antônio Carlos; SANTOS, Geraldo Tadeu dos. Produção e composição do leite, consumo de matéria seca e taxa de lotação em pastagens de gramíneas tropicais manejadas sob lotação rotacionada. Revista Brasileira de Zootecnia, v. 39, n.7, p. 1548-1557, 2010.

GERON, Luiz Juliano Valério; MEXIA, Alexandre Agostinho; GARCIA Jocilaine; SILVA Marciano Moreira da; ZEOULA, Lúcia Maria. Suplementação concentrada para cordeiros terminados a pasto sobre custo de produção no período da seca. Semina: Ciências Agrárias, Londrina, v. 33, p. 797-808, 2012.

GERON, Luiz Juliano Valério; MOURA, Daiane Caroline; RODRIGUES, Deivison Novaes; PAULA, Edson Júnior Heitor; TRAUTMANN-MACHADO, Raquel Joana; GARCIA, Jocilaine; SCHUMANN, Alline Mariá; SILVA; Dilma Alves. Viabilidade econômica de tourinhos terminados em confinamento alimentados com diferentes teores de caroço de algodão em dietas elaboradas com coprodutos agroindustriais. Semina: Ciências Agrárias, Londrina, v.35, p. 2673-2684, 2014.

GONÇALVES, Carlos Alberto; CAMARÃO, Ari Pinheiro; DUTRA, Saturnino; AZEVEDO, Guilherme Pantoja Calandrini de; MENDONÇA, Denise Castro; SOBRINHO, Carlos de Melo Junior. Produção de leite em pastejo rotacionado intensivo de "Brachiaria brizantha" cv. Marandu sob dois níveis de suplementação concentrado. In: Reunião anual da sociedade brasileira de zootecnia, 40., 2003, Santa Maria. Anais... Santa Maria: Sociedade Brasileira de Zootecnia, 2003.

IBGE -Instituto Brasileiro de Geografia e Estatística. [2018]. Pesquisa da pecuária nacional disponível em: <https://sidra.ibge.gov.br/Tabela/74>. Acessado em: 25 mar. 2020.

RC: 99522

Disponibile in: https://www.nucleodoconhecimento.com.br/veterinaria-it/pascolo-arotazione 
IPARDES: Instituto Paranaense de Desenvolvimento Econômico e Social. Caracterização sócio econômica da atividade leiteira no Paraná: Sumário executivo. Curitiba: IPARDES, 2009. 29 p.

JANK, Liana; ANDRADE, Carlos Mauricio Soares de; BARBOSA, Rodrigo Amorim; MACEDO, Manuel Claudio Motta; VALERIO, Jose Raul; VERZIGNASSI, Jaqueline Rosemeire; ZIMMER, Ademir Hugo; FERNANDES, Celso Dornelas; SANTOS, Mateus Figueiredo; RESENDE, Rosangela Maria Simeão. O capim-BRS Quênia (Panicum maximum Jacq.) diversificazione e intensificazione dei pascoli. Disponibile in: <https: ainfo.cnptia.embrapa.br/digital/bitstream/item/165106/1/capimbrs-quenia-panicum-maximum-jacq.pdf="'">.</https:> Accesso il: 25 mar. 2020.

MARION, Jose Carlos; SEGATTI, Sonia. Contabilidade da Pecuária. 9 ed. São Paulo: Atlas, 2010.

MARTIN, Nelson Batista; SERRA, Renata; OLIVEIRA, Marli Dias Mascarenhas; ÂNGELO, José Alberto; OKAWA, Hiroshige. Sistema "CUSTAGRI": sistema integrado de custos agropecuários. São Paulo: IEA/SAA, 1997. p. 1-75.

MARTINEZ, Junior Cesar. Produção de leite a pasto: manejo do pastejo é fundamental para manter a produção. 2009. Disponível em: $<$ http://www.milkpoint.com.br/radartecnico/nutricao/producao-deleite-a-pasto-manejodo-pastejo-e-fundamental-para-manter-aproducao-51710n.aspx>. Acesso em: 27 mai. 2020.

MATSUNAGA, Minoru; BEMELMANS, Paul Frans; TOLEDO, Paulo Edgard Nascimento de; DULLEY, Richard Domingues; HIROSHIGE, Okawa; PEDROSO, Iby Arvatti. Metodologia de custo utilizada pelo IEA. Agricultura em São Paulo, v. 23, n. 1, p.123-39, 1976.

OLIVEIRA, Jefferson Soares de. Análise de eficiência e alocação de recursos na produção leiteira do estado do Rio de Janeiro. In: congresso da sociedade

RC: 99522

Disponibile in: https://www.nucleodoconhecimento.com.br/veterinaria-it/pascolo-arotazione 
brasileira de economia, administração e sociologia rural, 45., 2007. Londrina. Conhecimentos para a agricultura do futuro: Anais... Brasília, DF: SOBER; Londrina: IAPAR: Universidade Estadual de Londrina, 2007.

PEREIRA, Lilian Elgalise Techio. et al. Recomendações para correção e adubação de pastagens tropicais. Pirassununga: Faculdade de Zootecnia e Engenharia de Alimentos da USP, 2018. 56p.

ROSESTOLATO, Lucas Luiz Rocha. et al. Viabilidade econômica comparativa de sistemas tecnológicos da pecuária leiteira no município de llha Solteira/SP. Congresso de extensão universitária da UNESP, 8., p. 1-6, 2015. Disponível em: <http://hdl.handle.net/11449/142491>. Acesso em: 28 mai. de 2020.

SEAB - Secretaria de Estado da Agricultura e do Abastecimento. Bovinocultura de leite: leite - $\quad$ produção 2016/17. $\quad$ Disponível em:<http://www.agricultura.pr.gov.br/arquivos/File/deral/Prognosticos/2018/leite_201 7_18.pdf>. Acesso em: 25 mar. de 2020.

Inserito: Luglio 2021.

Approvato: Settembre 2021.

RC: 99522

Disponibile in: https://www.nucleodoconhecimento.com.br/veterinaria-it/pascolo-arotazione 